\section{SLE and psychiatric morbidity}

SIR: I read with interest the study of Lim et al (Journal, October 1991, 159, 520-523). They reported a higher psychiatric morbidity among systemic lupus erythematosus (SLE) patients in Singapore compared with the controls and with SLE patients in London in another similar study by Lim et al (1988). However, the results must be interpreted with caution.

Firstly, the authors did not match the control group for age. As a result, the SLE group is significantly younger than the control. The observed difference may be compounded by the age factor. Younger patients facing chronic debilitating illness may suffer a greater psychiatric morbidity.

Secondly, selection of patients who speak English would have resulted in sampling a particular stratum of patients. In Singapore, most of the Chinese population speak Mandarin or one of the Southern Chinese dialects. The criterion would have excluded older and less-educated patients. This sample is therefore not representative of all SLE patients in Singapore. The authors may consider using a Chinese version of questionnaires and investigators wellversed in Chinese.

Thirdly, statistical computation using data provided by the authors shows that the difference in psychiatric morbidity between Singapore and London SLE patients is not statistically significant $\left(\chi^{2}=1.228, P>0.05,1\right.$ d.f. $)$.

Lim, L., Ron, M. A., et al (1988) Psychiatric and neurological manifestation in SLE. Quarterly Journal of Medicine (New Series 66), 249, 27-38.

Altman, D. G., el al (1983) Statistical guidelines for contributors to medical journals. British Medical Journal, 286, 1489-1493.

Department of Psychogeriatrics

S. H. ONG

St Mary Abbots Hospital

Marloes Road

London W8 $5 L Q$

\section{Disaster survivors}

SIR: Joseph et al (Journal, October 1991, 159, 542546) describe causal attributions and psychiatric symptoms in survivors of the Herald of Free Enterprise disaster. The study is interesting for its methods as well as its field of inquiry.

Obvious methodological problems are the small number of subjects, the selection of subjects, the exclusion of some from analysis after selection, the use of legal statements as measures of personal attributes, the use of correlation when regression analysis would be more informative and the assumption that correlation implies causation. Parametric statistical methods, which assume normal probability distributions, have been used for data which are clearly skewed (GHQ-30 and BDI scores: the mean is less than twice the standard deviation).

Further problems arise from the way in which the authors have processed their data to produce ratings for internality and controllability. They start with nominal data (e.g. the categories 'internal' 'external' and 'both internal and external') which they arbitrarily convert to ordinal data (e.g. categories ' $l$ '. '2' and ' 1.5 '). This is then processed to give discrete (discontinuous) numerical data (by adding together and taking the mean) which is analysed by methods appropriate only to continuous numerical data (Pearson's method of correlation).

Taking the internality score as an example, the total score for each subject will follow a binomial distribution. When a 'mean' is calculated, the distribution is scaled down to fit between the numbers 1 and 2. For example, with two 'negative-event' statements the only values that the internality rating can take are: $1,1.5$, and 2 . For four statements the values are: $1,1.25,1.5,1.75$ and 2 . The rating is not a continuous variable but can only have discrete numerical values.

Not only does this invalidate the method of analysis, but there is also a further difficulty. Where there is a small number of reported 'negative-events' a change in attribution of one event will have a very large effect on the internality rating. With a large number of reported events, a single change of attribution will have only a small effect on the rating. For example, with two events, a single change of attribution will cause the internality score to change by 0.5 but for ten events it will only change by 0.1 .

This would seem to invalidate non-parametric methods of analysis such as Spearman's rank correlation coefficient. Small changes in attribution score would cause large changes in rank for some observations.

When the overall internality or controllability rating is near to one of its extreme values, subjects with a low number of reported 'negative-events' will have a highly skewed distribution of rating values. Suppose that there is an overall probability of 0.08 of attributing a 'negative event' to the category 'uncontrollable'. (This is the overall probability given in the paper.) Then, by the binomial theorem, there is a probability of 0.15 of getting a final controllability score of 1.5 or greater when two 'negative-events' are reported. There is only a probability of 0.0012 of getting a score in this range when seven events are reported. When eight or more events are reported the probability is negligible. 
Most rating values will, therefore, be close to the mean but there is a high probability of one or more outlying points occurring. This will cause statistically significant, but spurious, correlations.

Taking all of this into consideration, I agree with the authors' conclusion that "Further research is clearly needed to substantiate these results".

Bill Plummer

Community Mental Health Centre

8, Radnor Park Avenue

Folkestone

Kent

\section{Prevalence and incidence of schizophrenia in Afro-Caribbeans}

SIR: The recent articles by Wessely et al (Journal, December 1991, 159, 795-801) and Castle et al (Journal, December 1991, 159, 790-794) describing increased incidence of schizophrenia in those of Afro-Caribbean origin, and Eagles' description of the possible alternatives to psychosocial explanations (Journal, December 1991, 159, 783-789) raise a number of issues.

A recent epidemiological study on the Caribbean island of Dominica showed that over a 30-month period the age-corrected prevalence rate for schizophrenia by ICD-9 diagnosis was $11.8 / 1000$ (Kay, 1990). This figure was obtained from a computerised case register of all admissions, out-patient referrals and prison consultations supplemented by the use of key informants in every major village. When DSMIII diagnostic criteria were applied, the prevalence decreased to 7.8/1000. Given the limitations of case finding in this study, the rates found by either diagnostic system are significantly higher than those generally found in the UK.

Incidence rates for schizophrenia in Dominica in 1989, using the same case register were, by ICD-9 $14.8 / 10000$, and using DSM-III criteria $7.3 / 10000$, which are considerably higher than comparative figures in Camberwell for the total population (Castle et al) by a factor of 6 to 8 , but closer to the increased risk ratio of schizophrenia if of AfroCaribbean origin (Wessely et $a$ ). The smaller proportion of ICD-9 cases meeting DSM-III criteria in Camberwell may reflect possible diagnostic bias and, in particular, cases of affective psychosis may masquerade as paranoid schizophreniform psychoses possibly as a result of culturally determined projective defence mechanisms being more prevalent in Afro-Caribbeans.

If results from one island state can be generalised to the Caribbean region this would suggest that the increased risk of schizophrenia meeting DSM-III criteria in the UK for those of Afro-Caribbean origin must be explained by factors operating both in the UK and the country of origin, and not the potentially racially prejudiced interaction with the UK mental health care system. Possible explanations include those factors discussed by Eagles. With increasing immunisation rates, and obstetric care approaching UK levels in Dominica (McIntyre, 1988), it will be interesting to observe if there is any decrease in the incidence of schizophrenia in the next 15 to 20 years. Increased genetic risk is possible but unlikely given low reported rates of schizophrenia in West Africa (Sikanertey \& Eaton, 1984) where most AfroCaribbeans originate, and theories based on concentration of genetic factors in small gene pools are unlikely given low rates of other congenital diseases.

A major culturally determined factor in my experience is that the consumption of large amounts of cannabis can act as both a precipitant of schizophreniform psychoses and as a factor in causing relapse and maintaining chronicity, in pre-existing schizophrenia, although the published literature is contradictory. This may partially explain the high incidence and prevalence rates found in Dominica if not to the same extent those found in Camberwell, where cannabis consumption may be assumed to be lower. However, this is an area requiring further study.

KAY, R. W. (1990) Prevalence of psychotic mental disorders in the Commonwealth of Dominica (Abstract) West Indian Medical Journal, 39 (suppl.1), 30-31.

MCINTYRE, D. O. N. (1988) In Annual Report of the Chief Medical Officer for the year 1987. Dominica: Ministry of Health.

SikANERTEY, T. \& Eaton, W. W. (1984) Prevalence of schizophrenia in the Labadi District of Ghana. Acta Psychiatrica Scandinavica 69, 156-161.

The Royal Edinburgh Hospital

RONALD W. KaY

Morningside Place

Edinburgh

EHIO 5HF

\section{Monsieur Pascal's cognitive therapy?}

SIR: I was intrigued recently to read the following and to note the similarities that there are with the concepts that underlie 'modern' cognitive therapies.

\footnotetext{
"The nature of man is so framed, that not only by often hearing himself a fool, he believes it; but by often calling himself a fool, he enters into the same opinion. Every person holds an inward and secret conversation with his own breast, and such as it highly concerns him well to regulate, because even in this sense, evil communications corrupt good manners. To study silence as much as possible and to converse with God alone, is the true persuasion in respect of ourselves."
} 